\section{PROTEIN-PROTEIN INTERACTIONS}

\section{Getting rid of JNK}

Jun N-terminal kinases (JNKs) phosphorylate and activate many cellular factors involved in disease processes, including diabetes, cancer, atherosclerosis and neurodegenerative diseases. As such, the potential of JNK inhibitors as therapeutics has attracted considerable interest. A key issue with kinase inhibitors, in particular with those that target the ATP-binding site, is target selectivity. Reporting in PNAS, Stebbins and colleagues seem to have overcome this problem as they have identified a novel and promising small-molecule JNK inhibitor that disrupts the interaction between JNK and its binding partner JNK interacting protein 1 (JIP1) both in vitro and in vivo.

The scaffolding protein JIP1 enhances JNK signalling by bringing JNK into proximity with its upstream kinases and substrates. Previous studies have demonstrated that expression of the JNK-binding domain of JIP1 acts as a competitive inhibitor of JNK signalling and restores normoglycaemia in a mouse model of type 2 diabetes. In this study, the authors screened 30,000 compounds for the ability to disrupt the interaction between JNK and JIP1 without causing cytotoxicity. They identified BI-78D3 as a potent inhibitor of this interaction and of JNK activity by using the DELFIA assay (dissociation enhanced lanthanide fluorescent immunoassay) and a time-resolved fluorescence energy transfer based kinase assay.

BI-78D3 displaced biotinylated JIP1 from GST-JNK1 and selectively inhibited JNK activity at nanomolar concentrations. Further characterization of the docked structure of BI-78D3 onto JNK1 revealed that the compound's benzodioxan, nitro and thiazole moieties are key to its inhibitory activity. Moreover, the interaction between BI-78D3 and JNK1 seems to be dependent on JNK R127, a residue that is known to be required for JIP1 binding, and C167.

In cells, BI-78D 3 was found to inhibit tumour-necrosis factor$\alpha$-induced phosphorylation of Jun, but no effect was found on its ability to phosphorylate ATF2, which is a substrate of both JNK and p38, confirming the selective action of the compound. Importantly, intravenous injection of BI-78D3 into mice with concanavalin A-induced liver damage, which has been shown to be JNK dependent, abrogated liver damage (as measured by a decrease in the release of alanine aminotransferase to the circulation). Similarly, BI-78D3 was able to restore insulin sensitivity in a mouse model of type 2 diabetes within a few hours.

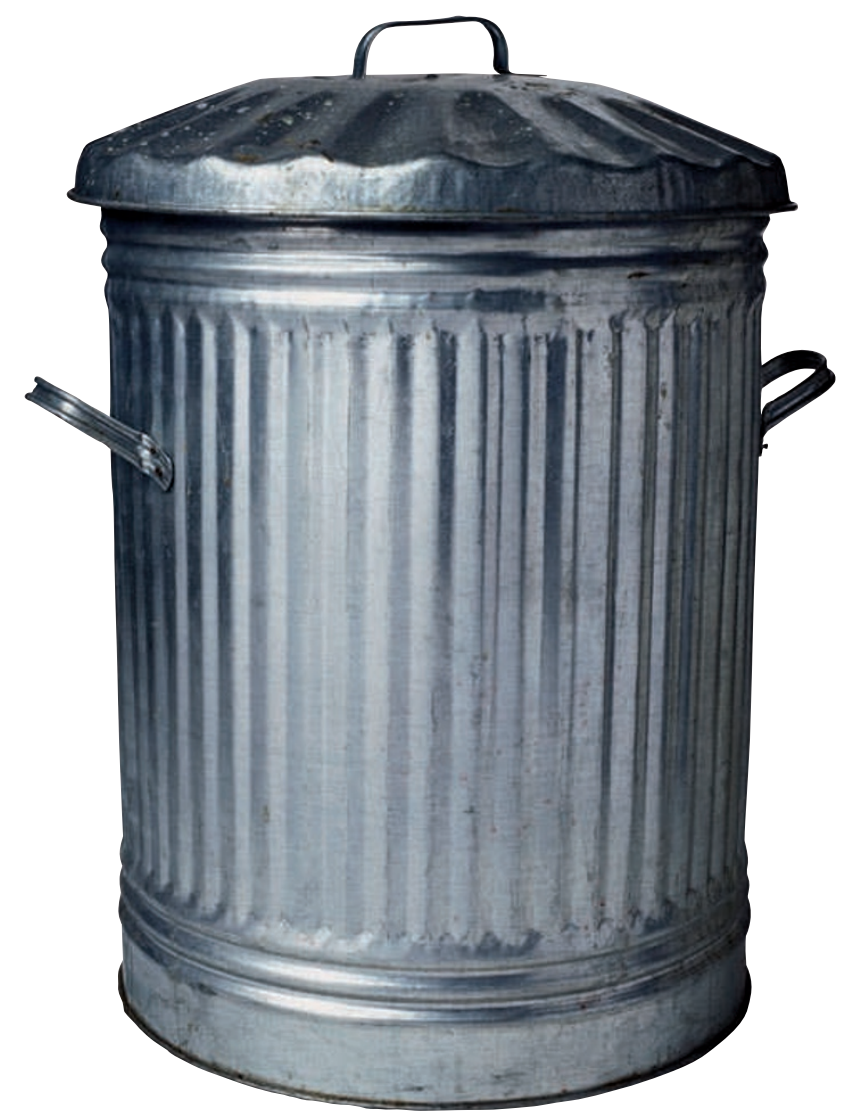

These results suggest that BI-78D3 is an effective JIP1 mimic that holds promise for the development of a new class of JNK inhibitors and highlight the potential of targeting the substrate docking sites of kinases, rather than the highly conserved ATP-binding sites, for therapeutic purposes.

Monica Hoyos Flight rather than the highly conserved ATP-binding sites.

ORIGINAL RESEARCH PAPER Stebbins, J. L. et al. Identification of a new JNK inhibitor targeting the JNK-JIP interaction site. Proc. Natl Acad. Sci. USA 105, 16809-16813 (2008) 